\title{
Integrated analysis of long non-coding RNA competing interactions reveals the potential role in progression of human gastric cancer
}

\author{
CHENG-YUN LI ${ }^{1}$, GE-YU LIANG ${ }^{1}$, WEN-ZHUO YAO ${ }^{1}$, JING SUI $^{1}$, XIAN SHEN $^{1}$, YAN-QIU ZHANG ${ }^{1}$, HUI PENG $^{1}$, \\ WEI-WEI HONG ${ }^{1}$, YAN-CHENG YE ${ }^{2}$, ZHI-YI ZHANG ${ }^{2}$, WEN-HUA ZHANG ${ }^{2}$, LI-HONG YIN ${ }^{1}$ and YUE-PU PU ${ }^{1}$ \\ ${ }^{1}$ Key Laboratory of Environmental Medicine Engineering, Ministry of Education, School of Public Health, \\ Southeast University, Nanjing, Jiangsu 210009; ${ }^{2}$ Gansu Wuwei Tumor Hospital, Wuwei, Gansu 733000, P.R. China
}

Received December 14, 2015; Accepted January 20, 2016

DOI: 10.3892/ijo.2016.3407

\begin{abstract}
Abnormal expression of long non-coding RNAs (lncRNAs) have been shown to play an important role in tumor biology. The Cancer Genome Atlas (TCGA) platform is a large sample sequencing database of lncRNAs, and further analysis of the associations between these data and patients' clinical related information can provide new approaches to find the functions of lncRNA. In the present study, 361 RNA sequencing profiles of gastric cancer (GC) patients were selected from TCGA. Then, we constructed the IncRNA-miRNA-mRNA competitive endogenous RNA (ceRNA) network of GC. There were 25 GC specific lncRNAs (fold change $>2, \mathrm{p}<0.05$ ) identified, 19 of them were included in ceRNA network. Subsequently, we selected these 19 key lncRNAs and analyzed the correlations with clinical features and overall survival, 14 of them were discriminatively expressed with tumor size, tumor grade, TNM stage and lymphatic metastasis $(\mathrm{p}<0.05)$. In addition, eight lncRNAs (RPLP0P2, FOXD2-AS1, H19, TINCR, SLC26A4-AS1, SMIM10L2A, SMIM10L2B and SNORD116-4) were found to be significantly associated with overall survival (log-rank $\mathrm{p}<0.05$ ). Finally, two key lncRNAs HOTAIR and UCA1 were selected for validation of their expression levels in 82 newly diagnosed GC patients by qRT-PCR. Results showed that the fold changes between TCGA and qRT-PCR were $100 \%$ in agreement. In addition, we also found that HOTAIR was significantly correlated with tumor size and lymphatic metastasis $(\mathrm{p}<0.05)$, and UCA1 was significantly correlated with tumor size, TNM stage and lymphatic metastasis $(\mathrm{p}<0.05)$.
\end{abstract}

Correspondence to: Professor Ge-Yu Liang, Key Laboratory of Environmental Medicine Engineering, Ministry of Education, School of Public Health, Southeast University, 87 Dingjiaqiao Street, Nanjing, Jiangsu 210009, P.R. China

E-mail: lianggeyu@163.com

Key words: gastric cancer, lncRNA, ceRNA network, clinical features, overall survival
The clinical relevance of the two lncRNAs and the bioinformatics analysis results were almost the same. Overall, our study showed the GC specific lncRNAs expression patterns and a ceRNA network in GC. Clinical features related to GC specific lncRNAs also suggested these lncRNAs are worthwhile for further study as novel candidate biomarkers for the clinical diagnosis of GC and potential indicators for prognosis.

\section{Introduction}

Noncoding RNAs (ncRNAs) are transcripts that have no ability of coding proteins, which widely exit in high eukaryotics. According to their characteristics, ncRNAs can be divided into several subtypes including transfer RNA, small nucleolar RNA (snoRNA), ribosomal RNA (rRNA), microRNA (miRNA) and long non-coding RNA (lncRNA). The amount of the ncRNAs transcripts is $>98 \%$ of the whole genome transcripts and have been suggested to represent transcriptional noise (1). However, more and more evidence indicates that transcriptional output of genome is far more complex than predicted, and suggests new paradigms of ncRNA regulation (2).

Recent studies suggest that the ncRNAs may play important biological roles in transcriptional regulation, cellular development, formation of chromosome and RNA modification (3). Based on the transcript size, ncRNAs are grouped into small ncRNAs (<200 bp) and long ncRNAs (>200 bp, up to $100 \mathrm{~kb}$ ). IncRNA is the functional end-product, and the level of lncRNA expression correlates directly with the level of the active molecule. Thus, the use of lncRNAs in diagnostics has intrinsic advantages over the use of protein-coding RNAs (4). In addition, IncRNAs show greater tissue specificity compared to miRNAs and protein-coding mRNAs, making them attractive in the search for novel diagnostic and prognostic cancer biomarkers (5). Increasing number of evidence shows that lncRNAs regulate the biological roles of various cancers in progression and development, including gastric cancer (GC) (6), esophageal cancer (7), hepatocellular carcinoma (HCC) (8), colorectal cancer (9), and lung cancer (10). According to the theory lncRNAs can regulate miRNAs and mRNAs by sequestering and binding them, many researchers 
also found lncRNA regulation of progression in GC $(11,12)$. In addition, cancer specific lncRNAs may also relate to invasion and metastasis of GC (13).

LncRNAs play important biological roles as a regulatory molecule through a variety of mechanisms. Salmena et al, presented the competing endogenous RNA (ceRNA) hypothesis, indicated that RNA transcripts communicate with each other by miRNA response elements (14). This competition between mRNAs, lncRNAs and pseudogene transcripts regulate each other's expression by using miRNA response elements (MREs) to compete for the binding of miRNAs, which exert an important role in the initiation and progression of tumor (15).

$\mathrm{Gu}$ et al (16), reported abnormal lncRNA expression profile of GC by microarray analysis from six GC patients. To date, there is also lack of large sample size studies and cancer specific lncRNA biomarkers or detection methods in GC. Moreover, small sample studies can not explain whether abnormal lncRNAs are related with gender, survival or other clinical features with statistical power. The Cancer Genome Atlas (TCGA) (http://cancergenome. nih.gov) project data portal provides a platform of RNA sequencing with mRNA, miRNA and IncRNA data for GC. To improve the reliability and accuracy of the results, we further explored lncRNAs in GC using data sets by the tools of TCGA. A total of 361 samples of GC tumor tissues, and 34 adjacent non-tumor stomach tissue RNA sequence results were included from TCGA. To the best of our knowledge, our study is the first to use the large scale sequencing database to explore the cancer specific lncRNA expression profiles and ceRNAs co-expression network in GC. We also used quantitative RT-PCR (qRT-PCR) validation for some of these bioinformatic analysis results in tumor tissues and adjacent non-tumor tissues from 82 newly diagnosed GC patients. This approach of finding cancer specific lncRNAs and ceRNA related network can help to clarify the functions of lncRNAs in GC.

\section{Materials and methods}

Patients and samples. A total of 443 patients with GC were collected from the TCGA database. The criteria of exclusion were set as follows: i) tissues samples without completed data for analysis; ii) histologic diagnosis is not stomach adenocarcinoma; iii) suffering of other malignancy except GC; iv) patients had received preoperative chemoradiation; and v) overall survival time more than 5 years. Overall, a total of $361 \mathrm{GC}$ patients were included in our study. Among these $361 \mathrm{GC}$ patients, the GC tumor tissues were obtained from 361 subjects and the adjacent non-tumor stomach tissues were from 34 subjects. In addition, there are 338 GC patients with lymphatic metastases and 23 GC patients with non-lymphatic metastases. According to the histologic staging data, well and moderately-differentiated adenocarcinoma GC (G1-2 stage) were 133 cases, and poorly-differentiated adenocarcinoma GC (G3-x stage) were 228 cases. This study was fully compliance with the publication guidelines provided by TCGA. The data were obtained by using TCGA database, so the approval of ethics committee was not required.
Table I. Differentially expressed intersection lncRNAs between G1-2 LM/Normal, G1-2 nLM/Normal, G3-x LM/Normal and G3-x nLM/Normal. ${ }^{\mathrm{a}}$

\begin{tabular}{|c|c|c|c|}
\hline $\begin{array}{l}\text { Name } \\
\text { (lncRNAs) }\end{array}$ & Transcript-ID & Regulation & $\begin{array}{c}\text { Average } \\
\text { fold-change }\end{array}$ \\
\hline HOXA11-AS & 221883 & Up & 16.58 \\
\hline HNF1A-AS1 & 283460 & Up & 13.09 \\
\hline HOTAIR & 100124700 & Up & 12.30 \\
\hline RPLP0P2 & 113157 & Up & 10.31 \\
\hline UCA1 & 652995 & Up & 9.29 \\
\hline PVT1 & 5820 & Up & 7.34 \\
\hline H19 & 283120 & Up & 6.66 \\
\hline FOXD2-AS1 & 84793 & Up & 6.15 \\
\hline GUCY1B2 & 2974 & Up & 4.84 \\
\hline LOC553137 & 553137 & Up & 4.63 \\
\hline TSPEAR-AS2 & 114043 & Up & 3.86 \\
\hline FCGR1C & 100132417 & Up & 3.82 \\
\hline ATP8B5P & 158381 & Up & 3.43 \\
\hline IGF2BP2-AS1 & 646600 & Up & 3.34 \\
\hline LOC100131496 & 100131496 & Up & 3.28 \\
\hline RHPN1-AS1 & 78998 & Up & 3.13 \\
\hline PGM5-AS1 & 572558 & Down & -11.98 \\
\hline PART1 & 25859 & Down & -11.59 \\
\hline SNORD116-4 & 100033416 & Down & -5.71 \\
\hline LOC100128164 & 100128164 & Down & -5.06 \\
\hline SMIM10L2A & 399668 & Down & -4.60 \\
\hline SLC26A4-AS1 & 286002 & Down & -3.57 \\
\hline GGTA1P & 2681 & Down & -3.36 \\
\hline SMIM10L2B & 644596 & Down & -3.36 \\
\hline TINCR & 257000 & Down & -3.28 \\
\hline
\end{tabular}

a25 GC-specific lncRNAs for ceRNA network construction with absolute fold change $>2.0, \mathrm{p}<0.05$.

In addition, 82 GC patient specimens, aged 45-70 years, and their paired adjacent non-cancerous tissue specimens were obtained from the Wuwei Tumor Hospital of Gansu (Wuwei, China), for quantitative RT-PCR analysis. All of these patients were assigned a diagnosis of GC based on histopathology and clinical history. Clinical information that was recorded for each specimen included age, tumor grade, cancer stage, tissue dimensions, and date of resection. None of the patients had received preoperative chemoradiation. Adjacent noncancerous tissues were located $\geq 5 \mathrm{~cm}$ from the tumor edge. Tissue samples were immersed in RNAlater (Ambion, Austin, TX, USA) and stored at $-80^{\circ} \mathrm{C}$ until use.

RNA sequence data sets and differential analysis. The stomach adenocarcinoma (STAD) RNA expression profile data (level 3) of the corresponding patients were obtained from TCGA data portal (up to Nov 1, 2015) (https://tcga-data. nci.nih.gov/tcga/dataAccessMatrix.htm?mode=ApplyFilter). TCGA database provide the normalized count data of RNA sequencing including lncRNA and mRNA expression 


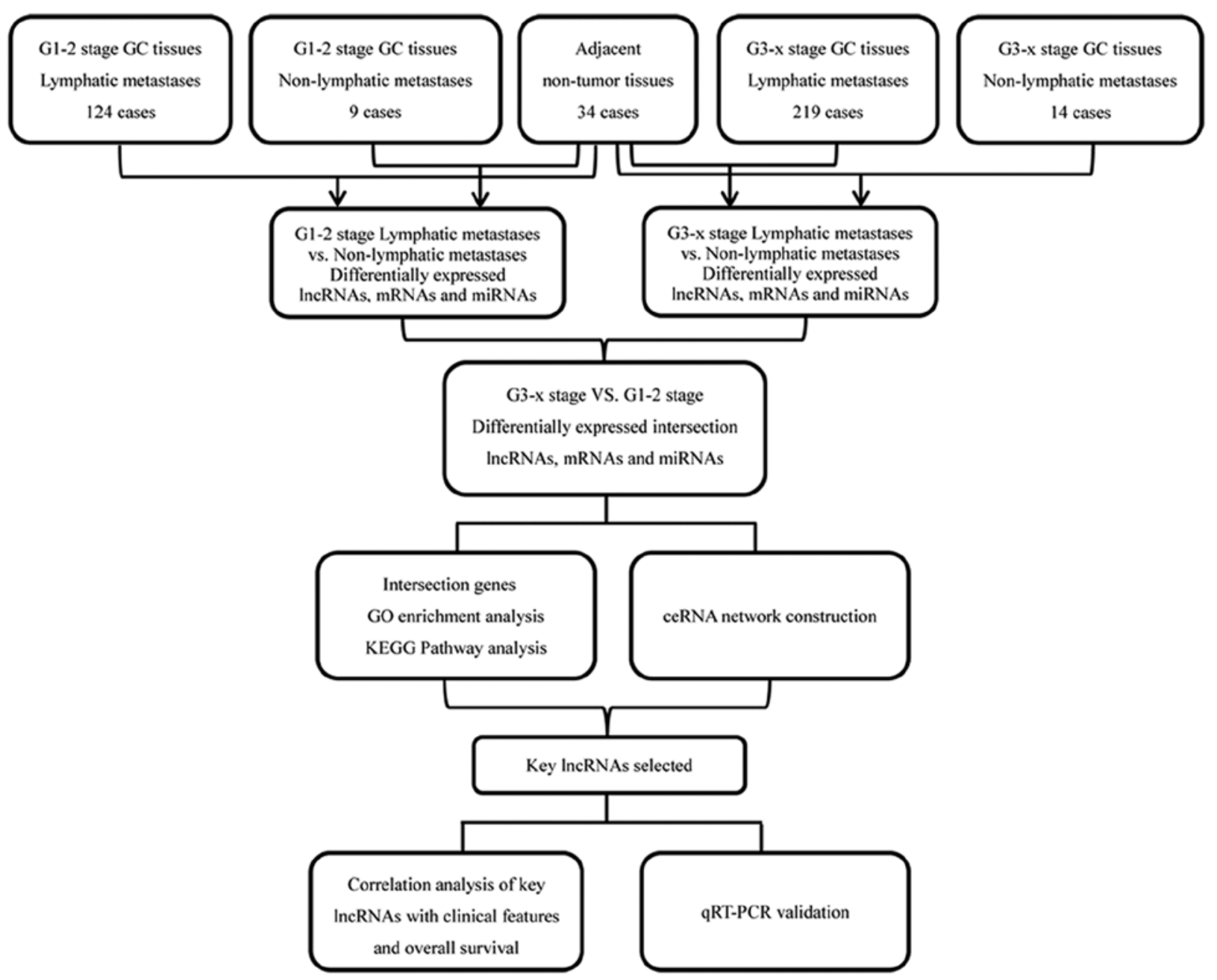

Figure 1. Flow chart of bioinformatics analysis.

profiles by RNASeqV2 system. The STAD level 3 microRNA sequencing (miRNAseq) data, downloaded from TCGA database, were collected by Illumina HiSeq 2000 microRNA sequencing platforms (Illumina Inc., Hayward, CA, USA). The downloaded data including many individual data files consist of one for each tissue sample. TCGA database have already normalized these RNA expression profile data, so no further normalization was required. In the next step, we compared the differentially expressed lncRNAs, mRNAs and miRNAs in 3 levels, including, GC patient tumor tissues/ adjacent non-tumor stomach tissues, lymphatic metastases of GC patients/non-lymphatic metastases of GC patients, well and moderately-differentiated (G1-2 stage) adenocarcinoma of GC/poorly-differentiated (G3-x stage) adenocarcinoma of GC, respectively. Then, we chose intersection IncRNAs, mRNAs and miRNAs in the above 3 levels for further analysis. Fig. 1 depicts a flow chart for bioinformatics analysis.

GO and Pathway analysis. Differentially expressed intersection mRNAs were entered into the Gene Ontology database (http://www.geneontology.org), which utilized GO to identify the molecular function represented in the gene profile. Up and downregulated genes were analyzed, respectively. The Kyoto Encyclopedia of Genes and Genomes (KEGG) (http://www. kegg.jp/) was used to analyze the potential functions of these genes participated in the pathways (17).

Construction of the ceRNA network. According to the relationship among IncRNA, miRNA and mRNA, the posttranscriptional regulation of mRNA transcripts bound by single-stranded miRNAs is basically established. In this study, we built miRNA-lncRNA-mRNA ceRNA network which are based on the theory that IncRNA can regulate miRNA abundance by sequestering and binding them, acting as so-called miRNA sponges. We chose differentially expressed intersection miRNA, IncRNA, and mRNA with fold change $>2.0$ (including upregulation and downregulation) and $\mathrm{p}<0.05$. Predicted differentially expressed miRNA targets in this study were determined using miRanda (http:// www.microrna.org/microrna/home.do) to find the IncRNAmiRNA interactions, and using mRBase targets (http://mirdb. org/miRDB/) and Targetscan (http://www.targetscan.org/) to predict target genes. Next the study combined the information of miRNAs predicted and differentially expressed data of TCGA to choose the intersection lncRNAs and mRNAs. Then, according to the theory of ceRNA, we chose the miRNA negatively regulated intersection expression of lncRNAs and mRNAs to construct the ceRNA network. 


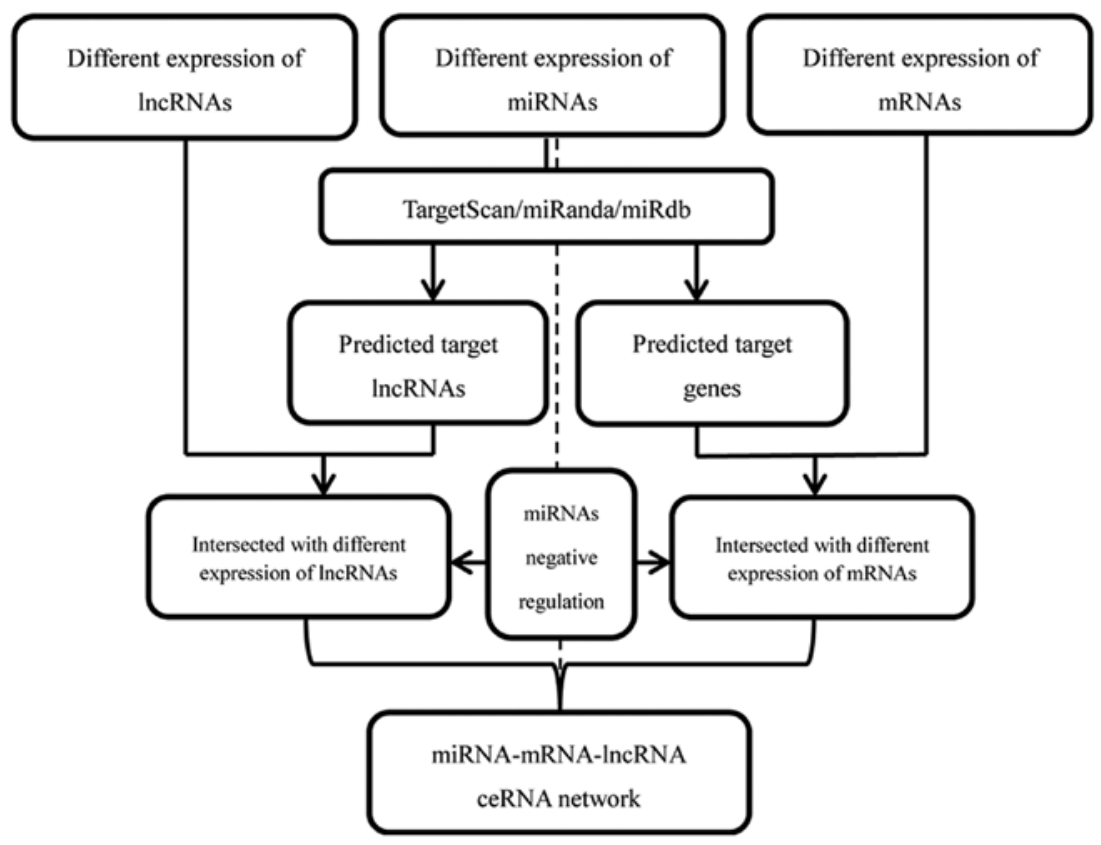

Figure 2. Flow chart of miRNA-lncRNA-mRNA network analysis.

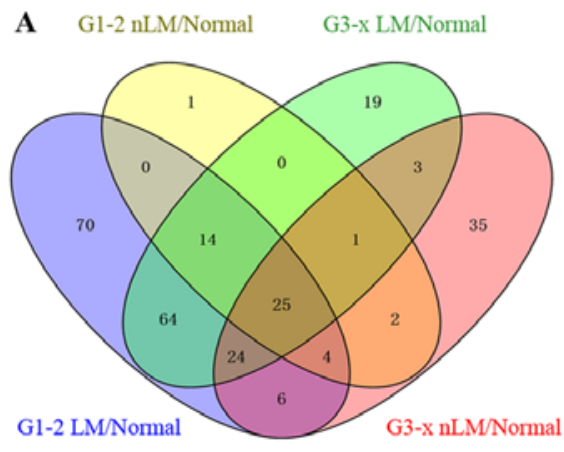

IncRNA

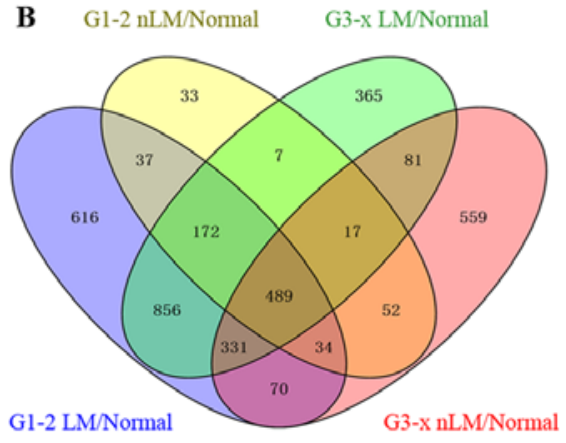

mRNA

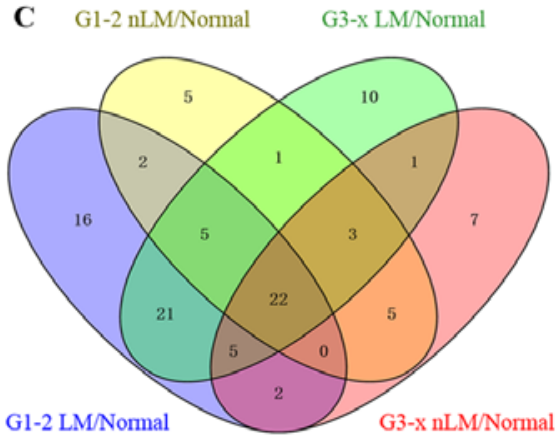

miRNA

Figure 3. Venn diagram analysis of differentially expressed (A) lncRNAs, (B) mRNAs and (C) miRNAs between G1-2 LM/Normal, G1-2 nLM/Normal, G3-x LM/Normal and G3-x nLM/Normal. LM, lymphatic metastasis; nLM, non-lymphatic metastasis; Normal represent adjacent non-tumor tissues.

The ceRNA networks were constructed and visualized using Cytoscape v3.0 (18). Fig. 2 depicts a flow chart for ceRNA network construction.

Key lncRNAs and clinical feature analysis and $q R T-P C R$ validation. According to the bioinformatics analysis and the ceRNA network, key lncRNAs were chosen. We further analyzed the clinical features including race, gender, tumor grade, TNM stage, pathological stage and invasion. In addition, our study also analyzed the association between the key lncRNAs and the GC patient survival time. Finally, we selected some of the key lncRNAs and used qRT-PCR to validate the results of bioinformatics analysis from 82 newly diagnosed GC patients.

Statistical analysis. All the results were expressed as mean \pm SD. Statistical analysis was done with Student's t-test for comparison of two groups in data analysis, and ANOVA for multiple comparisons. In both cases, differences with $\mathrm{p}<0.05$ were considered statistically significant. The statistical significance of data analysis results was analyzed by fold change and Student's t-test. False discovery rate was calculated to correct the p-value. qRT-PCR relative fold change results are calculated using the $2^{-\Delta \Delta \mathrm{Ct}}$ method (19), where $\left[\Delta \mathrm{Ct}=\left(\mathrm{Ct}_{\mathrm{RNAs}}-\mathrm{Ct}_{\mathrm{GAPDH}}\right)\right.$ and $\Delta \Delta \mathrm{Ct}=\Delta \mathrm{Ct}$ tumor tissues $-\Delta \mathrm{Ct}$ adjacent non-tumor tissues $]$.

\section{Results}

Cancer specific lncRNAs in GC. We identified that 268 lncRNAs were differentially expressed between GC tumor tissues and adjacent non-tumor tissues from TCGA database (absolute fold change $>2, p<0.05$ ). In further analysis of these differentially expressed lncRNAs, between GC tumor tissues and adjacent non-tumor tissues, we found 208 lncRNAs were differentially expressed between G1-2 stage (lymphatic metastasis) GC tumor tissues and adjacent non-tumor tissues; 

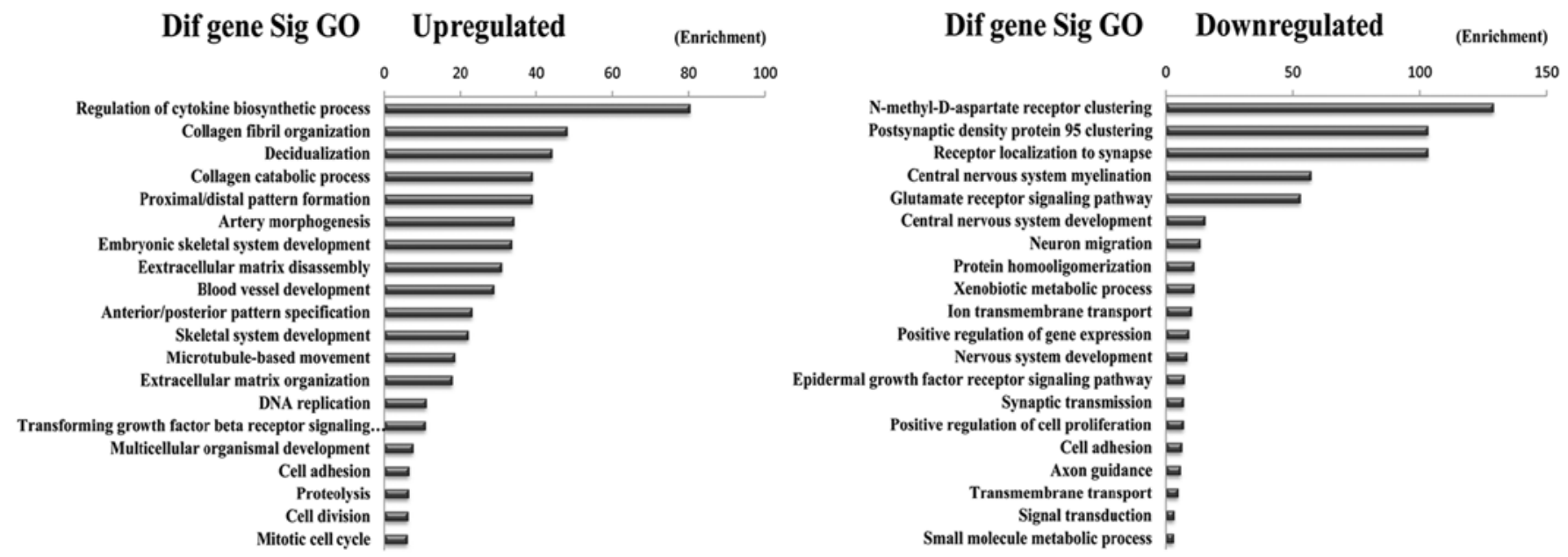

Figure 4. Top 20 enrichment of GO terms for differentially expressed intersection mRNAs (the bar plot shows the enrichment scores of the significant enrichment GO terms).
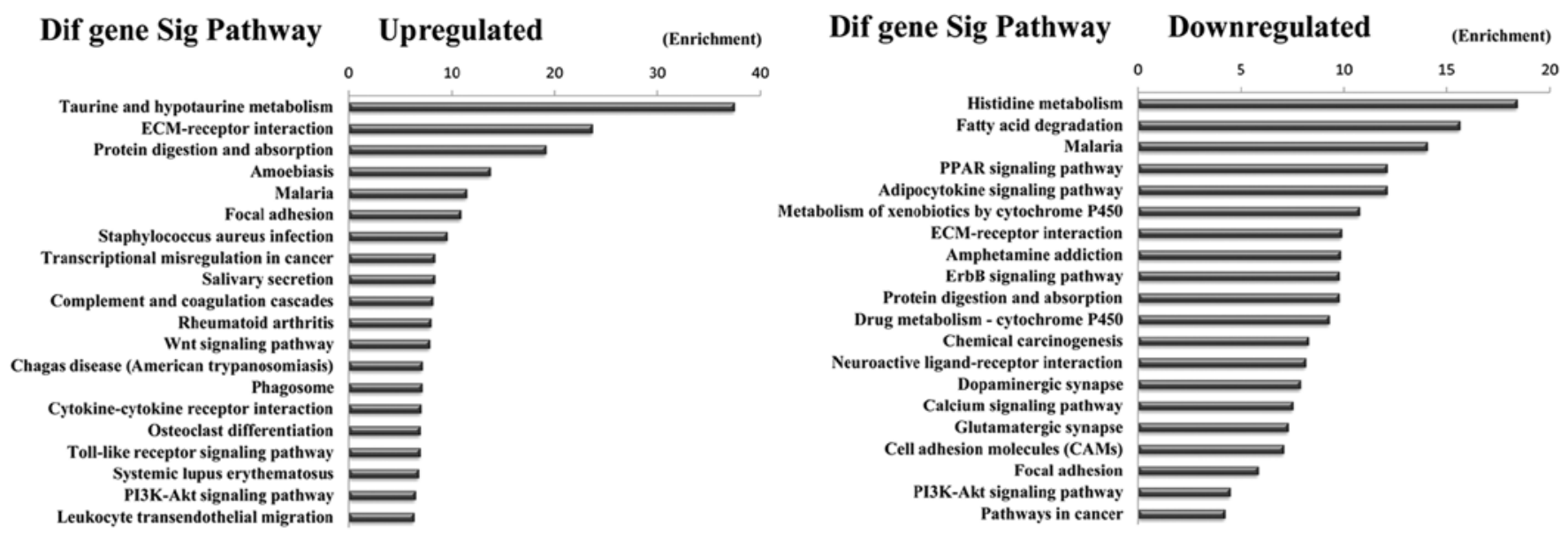

Figure 5. Top 20 enrichment of pathways for differentially expressed intersection mRNAs (the bar plot shows the enrichment scores of the significant enrichment pathways).

48 lncRNAs were differentially expressed between G1-2 stage (non-lymphatic metastasis) GC tumor tissues and adjacent non-tumor tissues; 153 lncRNAs were differentially expressed between G3-x stage (lymphatic metastasis) GC tumor tissues and adjacent non-tumor tissues; 101 lncRNAs were differentially expressed between G3-x stage (non-lymphatic metastasis) GC tumor tissues and adjacent non-tumor tissues. To further enhance the reliability of the bioinformatics analysis, we selected 25 lncRNAs included in the intersection of the above analyzed levels (Fig. 3A). Finally, 25 lncRNAs (16 upregulated; 9 downregulated) were selected to build the ceRNA network (Table I).

GO and Pathway analysis of differentially expressed genes. Predicted functions of differentially expressed genes in this study were determined by intersection mRNAs. Our study found that $3719 \mathrm{mRNAs}$ were differentially expressed between GC tumor tissues and adjacent non-tumor tissues in TCGA. We further analyzed these differentially expressed genes and found 2605 mRNAs were differentially expressed between G1-2 stage (lymphatic metastasis) GC tumor tissues and adjacent non-tumor tissues; 841 mRNAs were differentially expressed between G1-2 stage (non-lymphatic metastasis) GC tumor tissues and adjacent non-tumor tissues; 2318 mRNAs were differentially expressed between G3-x stage (lymphatic metastasis) GC tumor tissues and adjacent non-tumor tissues; 1633 mRNAs were differentially expressed between G3-x stage (non-lymphatic metastasis) GC tumor tissues and adjacent non-tumor tissues. Finally, we selected 489 mRNAs, which included in the intersection of the above analyzed levels (Fig. 3B).

The 489 differentially expressed genes were further analyzed by Gene Ontology database (http://www.geneontology.org). The upregulated and down regulated genes were analyzed, respectively. We analyzed the enrichment of these differentially expressed genes. Enrichment provides a measure of the significance of the function, and as the enrichment increases, the corresponding function is more specific, which helps us to identify GO with a more definitive functional description (20). The results showed that the highest enriched GOs targeted by upregulated transcripts were 'Regulation of cytokine biosynthetic processes'. The highest enriched GOs targeted by downregulated transcripts were ' $\mathrm{N}$-methyl-Daspartate receptor clustering' (Fig. 4). 
Table II. miRNAs targeting specific intersection key lncRNAs in GC.

\begin{tabular}{|c|c|}
\hline Key lncRNAs & miRNAs \\
\hline ATP8B5P & miR-133a-3p, miR-133b, miR-145-3p, miR-204-5p, miR-30c-2-3p \\
\hline FOXD2-AS1 & miR-129-5p, miR-139-5p, miR-145-3p, miR-145-5p \\
\hline GUCY1B2 & miR-129-5p, miR-145-3p, miR-145-5p, miR-204-5p, miR-30c-2-3p \\
\hline H19 & miR-129-5p, miR-145-3p, miR-145-5p, miR-486-5p \\
\hline HOTAIR & miR-1, miR-133a-3p, miR-133b, miR-145-3p, miR-204-5p \\
\hline LOC 100128164 & miR-182-5p, miR-183-5p, miR-194-5p, miR-196b-5p, miR-335-3p, miR-4326 \\
\hline SNORD116-4 & miR-1, miR-139-3p, miR-204-5p, miR-30c-2-3p, miR-363-3p \\
\hline LOC553137 & miR-129-5p, miR-139-5p, miR-144-5p, miR-145-5p, miR-30c-2-3p \\
\hline PART1 & miR-135b-5p, miR-182-5p, miR-196a-5p, miR-196b-5p, miR-335-3p, miR-4326 \\
\hline PGM5-AS1 & miR-182-5p, miR-335-3p, miR-4326 \\
\hline PVT1 & $\operatorname{miR}-30 c-2-3 p$ \\
\hline RHPN1-AS1 & miR-145-3p, miR-145-5p, miR-451a, miR-486-5p \\
\hline RPLP0P2 & miR-129-5p, miR-30c-2-3p \\
\hline SLC26A4-AS1 & miR-194-5p, miR-335-3p \\
\hline SMIM10L2A & miR-182-5p, miR-183-5p, miR-194-5p, miR-196a-5p, miR-196b-5p, miR-4326 \\
\hline SMIM10L2B & miR-182-5p, miR-196a-5p, miR-196b-5p, miR-4326 \\
\hline TINCR & miR-135b-5p, miR-196a-5p, miR-196b-5p, miR-4326 \\
\hline TSPEAR-AS2 & miR-144-5p, miR-486-5p \\
\hline UCA1 & $\operatorname{miR}-1$ \\
\hline
\end{tabular}

Pathway analysis indicated that 23 pathways corresponded to upregulated transcripts and that the most enriched network was 'Taurine and hypotaurine metabolism'. Moreover, pathway analysis also showed that 39 pathways corresponded to downregulated transcripts and that the most enriched network was 'Histidine metabolism'. Among these pathways, such as the 'PI3K-Akt signaling pathway', is involved in the carcinogenesis of gastric adenocarcinoma metastases (21), the 'Wnt signaling pathway' has been investigated as a cause of adenocarcinoma invasion (22) and the gene category 'Pathways in cancer' is involved in the development of GC. In addition, some pathways such as 'Small cell lung cancer, Cell adhesion molecules (CAMs), Proteoglycans in cancer and Transcriptional regulation in cancer' were also reported as cancers related pathways $(23,24)$ (Fig. 5).

Predicted miRNAs targeted relationship and ceRNA network construction. In this study we have found $105 \mathrm{GC}$ associated miRNAs which were differentially expressed between GC tumor tissues and adjacent non-tumor tissues. We selected 22 intersection miRNAs from 105 GC associated miRNAs by bioinformatics analysis from G1-2 lymphatic metastases GC patients /non-lymphatic metastases GC patients and G3-X lymphatic metastases GC patients /non-lymphatic metastases GC patients (Fig. 3C). In the next step, we focused on whether these intersection miRNAs would target the above $25 \mathrm{GC}$ specific lncRNAs. In the ceRNAs network, 17 miRNAs targeted 19 key lncRNAs were predicted though miRcode (http://www.mircode.org/) (25) (Table II).

Then, in order to establish the IncRNA-miRNA-mRNA ceRNAs network, we also search for mRNAs targeted by miRNAs. According to the miRNAs described in Table II,
Table III. miRNAs targeted cancer specific intersection mRNAs.

\begin{tabular}{|c|c|}
\hline miRNAs & mRNAs \\
\hline miR-1 & ADAM12, CELSR3, FN1 \\
\hline $\operatorname{miR}-129-5 p$ & $\begin{array}{l}\text { COL1A1, CSMD2, EME1, AKT3, } \\
\text { HOXC13, HOXC8, IGF2BP1, } \\
\text { KIT, PMEPA1, SALL4 }\end{array}$ \\
\hline miR-133a-3p & XIRP1 \\
\hline $\operatorname{miR}-135 b-5 p$ & $\begin{array}{l}\text { COX6B2, ERBB4, GHR, GPR155, MAOB, } \\
\text { GRIK3, HDC, MYOCD, PAK7, PDE8B, } \\
\text { PLCXD3, PRIMA1, RIMS1, SLITRK6 }\end{array}$ \\
\hline miR-139-5p & HOXA9, KIT \\
\hline $\operatorname{miR}-145-5 p$ & SERPINE1, TNFR, SF11B \\
\hline $\operatorname{miR}-182-5 p$ & $\begin{array}{l}\text { ATOH8, CADM2, CHL1, BIRC7, ASPA, } \\
\text { FAM107A, FIGF, MAOB, GRIK3, KLF15, } \\
\text { LHFPL4, MYRIP, PDK4, RIMS3, SLC16A9 }\end{array}$ \\
\hline $\operatorname{miR}-183-5 p$ & COMP \\
\hline miR-194-5p & AFF3, GRIN2A, ATM \\
\hline miR-196a-5p & ACSL6, AQP4, NRXN1, OPCML, RSPO2 \\
\hline $\operatorname{miR}-204-5 p$ & ADAM12, CELSR3, HMGA2, HOXC8, KIT \\
\hline $\operatorname{miR}-30 c-2-3 p$ & AKT3, IGF2BP1, TOP2A \\
\hline $\operatorname{miR}-335-3 p$ & $\begin{array}{l}\text { ACSL6, GHR, GPR155, GRIN2A, HS6ST3, } \\
\text { COL4A5, COL4A6, KIAA2022, LIFR, } \\
\text { MYRIP, PARK2, PLCXD3, COL11A6, ATP2B }\end{array}$ \\
\hline $\operatorname{miR}-363-3 p$ & HMGA2, NOX4, ZNF469 \\
\hline
\end{tabular}

we predicted miRNAs targeted mRNAs though miRTarBase (http://mirtarbase.mbc.nctu.edu.tw/) (26). Finally, we selected 

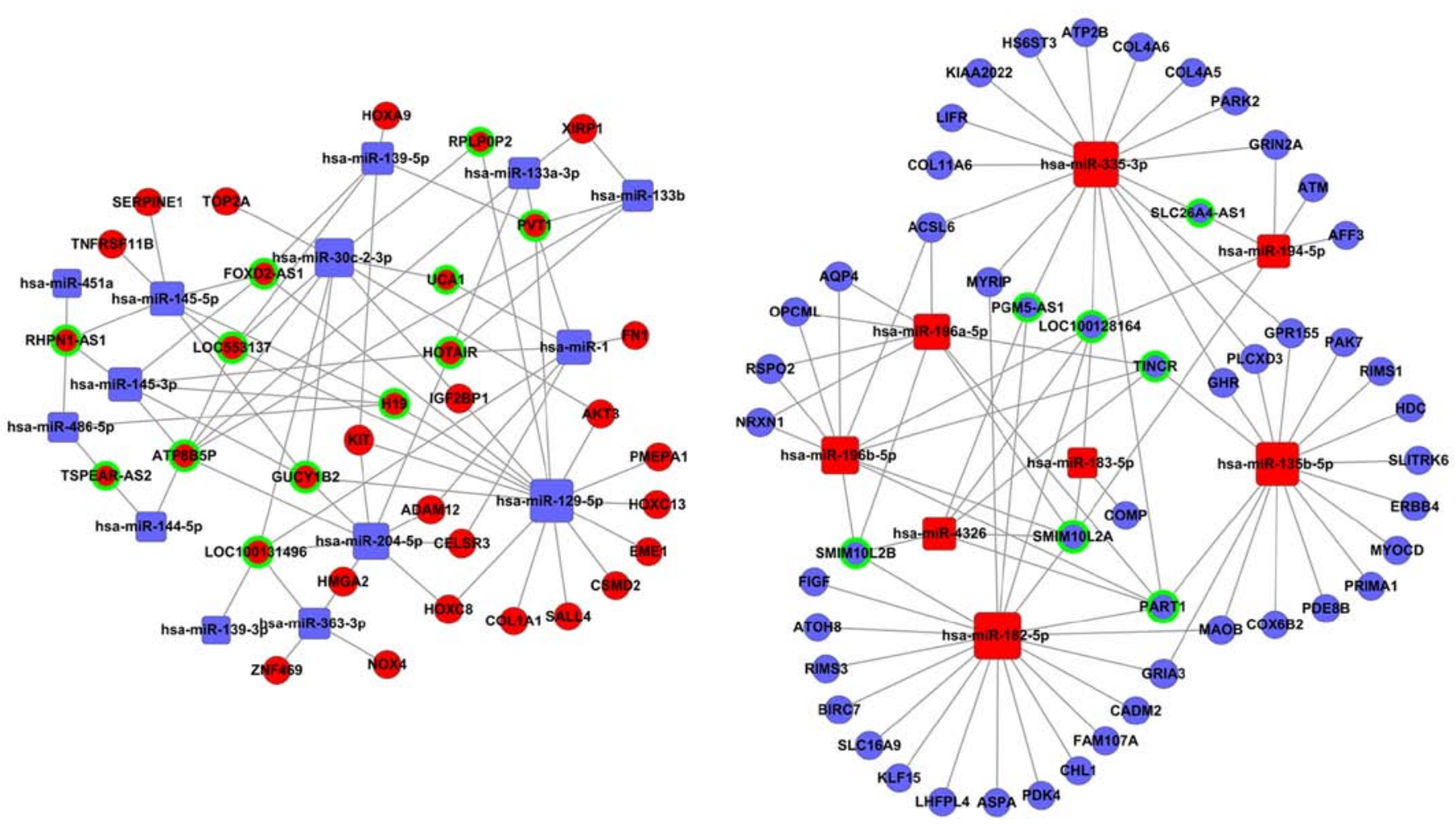

Figure 6. The lncRNA-miRNA-mRNA ceRNA network. Red diamonds represent upregulated miRNAs, red balls, upregulated mRNAs, and red balls surrounded by green rings, upregulated lncRNAs. Blue diamonds represent downregulated miRNAs, blue balls, downregulated mRNAs; blue balls surrounded by green rings, downregulated lncRNAs.

the intersection mRNAs through the predicted mRNAs and bioinformatics analyzed differentially expressed 489 mRNAs. The results identified 14 miRNAs (Table III) related to the 67 intersection mRNAs, and some of them have been reported to be cancer-associated genes such as COL1A1, FIGF, GHR, HOXC10, HOXC13, HOXC8, LIFR and SF11B.

Based on Tables II and III, we constructed a miRNA-lncRNA-mRNA ceRNA network. The miRNAlncRNA-mRNA relationship was integrated into the ceRNA network through negative regulation. The ceRNA network was drawn using Cytoscape 3.0. Nineteen lncRNAs, 22 miRNAs (Tables II and III complementary miRNAs) and 67 mRNAs were involved in the ceRNA network (Fig. 6). We also analyzed the mRNAs involved in ceRNA network to understand the IncRNAs indirectly regulated signal pathways by DAVID database (https://david.ncifcrf.gov/). According to the number of mRNAs involved, we listed the top 8 KEGG pathways in our study (Table IV). Four cancer-related pathways including pathways in 'Pathways in cancer, Small cell lung cancer, Renal cell carcinoma and PI3K-Akt signaling pathway' were enriched with the mRNAs, another 4 non-cancer related pathways such as 'Focal adhesion, ECM-receptor interaction, Histidine metabolism and ErbB signaling pathway' were also enriched.

Key lncRNAs and clinical feature association and qRT-PCR validation. The 19 key lncRNAs from the ceRNA network were further analyzed according to the clinical features, respectively, including race, gender, tumor grade, TNM stage and lymphatic metastasis status in TCGA data sets. There were 14 GC specific lncRNAs, the expression levels of which were significantly different in comparison of clinical features
Table IV. KEEG pathways enriched by the coding genes involved in the ceRNA network.

\begin{tabular}{|c|c|}
\hline KEEG pathways & Genes \\
\hline \multicolumn{2}{|l|}{ Cancer related } \\
\hline Pathways in cancer & $\begin{array}{l}\text { KIT, FIGF, COL4A6, } \\
\text { AKT3, FN1 }\end{array}$ \\
\hline Small cell lung cancer & COL4A6, AKT3, FN1 \\
\hline Renal cell carcinoma & PAK7, FIGF, AKT3 \\
\hline PI3K-Akt signaling pathway & AKT3, KIT, FIGF \\
\hline \multicolumn{2}{|l|}{ Non-cancer related } \\
\hline Focal adhesion & $\begin{array}{l}\text { PAK7, COMP, COL1A1, } \\
\text { FIGF, COL4A6, AKT3, FN1 }\end{array}$ \\
\hline ECM-receptor interaction & $\begin{array}{l}\text { COMP, COL1A1, COL4A6, } \\
\text { FN1 }\end{array}$ \\
\hline Histidine metabolism & ASPA, HDC, MAOB \\
\hline ErbB signaling pathway & PAK7, ERBB4, AKT3 \\
\hline
\end{tabular}

$(\mathrm{p}<0.05)$. We found that ATP8B5P, FOXD2-AS1, UCA1, GUCY1B2, RHPN1-AS, TSPEAR-AS2, LOC100128164 and SLC26A4-AS1 were linked to tumor grade, PVT1, H19 and PART1 were linked to TNM stage, LOC553137, HOTAIR and TINCR were linked to lymphatic metastasis (Table V).

Furthermore, to further identify the 19 key lncRNAs with prognostic characteristics from 361 GC patients, the lncRNA data set and the overall survival information were profiled by the univariate Cox proportional hazards regression 

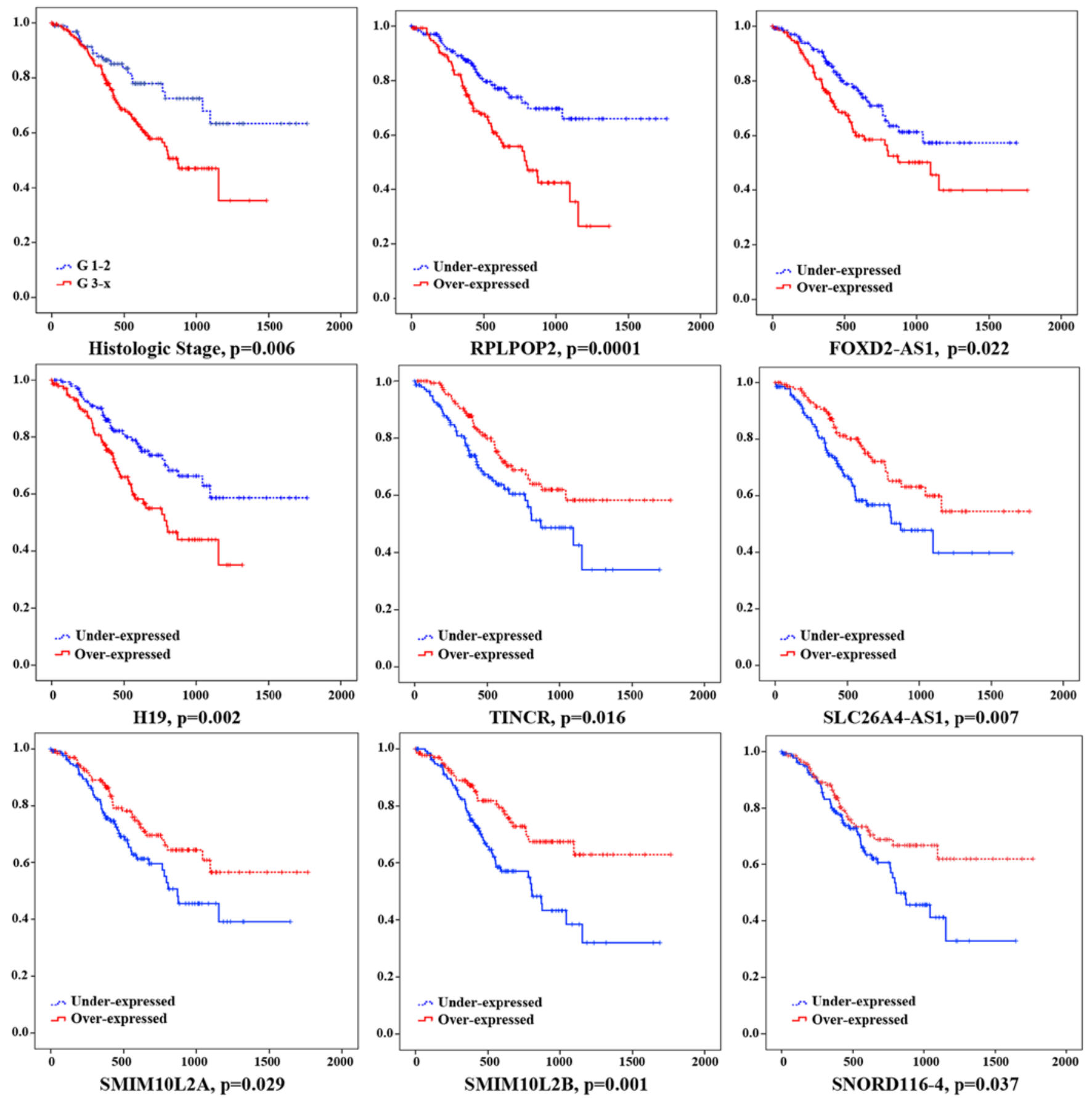

Figure 7. Kaplan-Meier survival curves for eight lncRNAs associated with overall survival. Horizontal axis: overall survival time, days; Vertical axis: survival function.

model and 8 lncRNAs were found significantly associated with GC patients' overall survival (log-rank $\mathrm{p}<0.05)$. Among the 8 significant lncRNAs, 3 lncRNA (RPLP0P2, FOXD2-AS1 and H19) were negatively associated with overall survival $(\mathrm{p}<0.05)$, while the remaining 5 lncRNA (TINCR, SLC26A4-AS1, SMIM10L2A, SMIM10L2B and SNORD116-4) were positively correlated with overall survival (p<0.05) (Fig. 7).

Subsequently, to confirm the reliability and validity of the above analyzed results, we random selected 2 key lncRNAs (HOTAIR, UCA1) and analyzed their actual expression levels in 82 newly diagnosed tumor tissues of GC patients and adjacent non-tumor tissues. Results suggest that HOTAIR and UCA1 both were significantly higher expressed in GC tumor tissues than adjacent non-tumor tissues. The results from the qRT-PCR validation in 82 newly diagnosed GC patients and the above bioinformatics results (Table I) were $100 \%$ in agreement (Fig. 8). To further analyze the association between the 2 key lncRNAs and clinicopathological characteristics of $82 \mathrm{GC}$ patients, we found that HOTAIR was significantly associated with tumor size and lymphatic metastasis, and UCA1 was significantly associated with tumor size, TNM stage and lymphatic metastasis (Fig. 9). The two lncRNAs are clinically relevant and the results of bioinformatics analysis were almost 
Table V. The correlation between cancer specific lncRNAs and clinical features.

\begin{tabular}{lll}
\hline Comparisons & \multicolumn{1}{c}{ Upregulated } & Downregulated \\
\hline Gender (Female vs. Male) & & TINCR \\
Race (White vs. Asian) & H19 & \\
Tumor grade (G3-x vs. G1-2) & ATP8B5P, FOXD2-AS1, UCA1, GUCY1B2, & LOC100128164, \\
& RHPN1-AS, TSPEAR-AS2 & SLC26A4-AS1 \\
TNM staging system (T3 + T4 vs. T1 + T2) & PVT1, H19 & PART1 \\
Lymphatic metastasis (No vs. Yes) & LOC553137, HOTAIR & TINCR
\end{tabular}

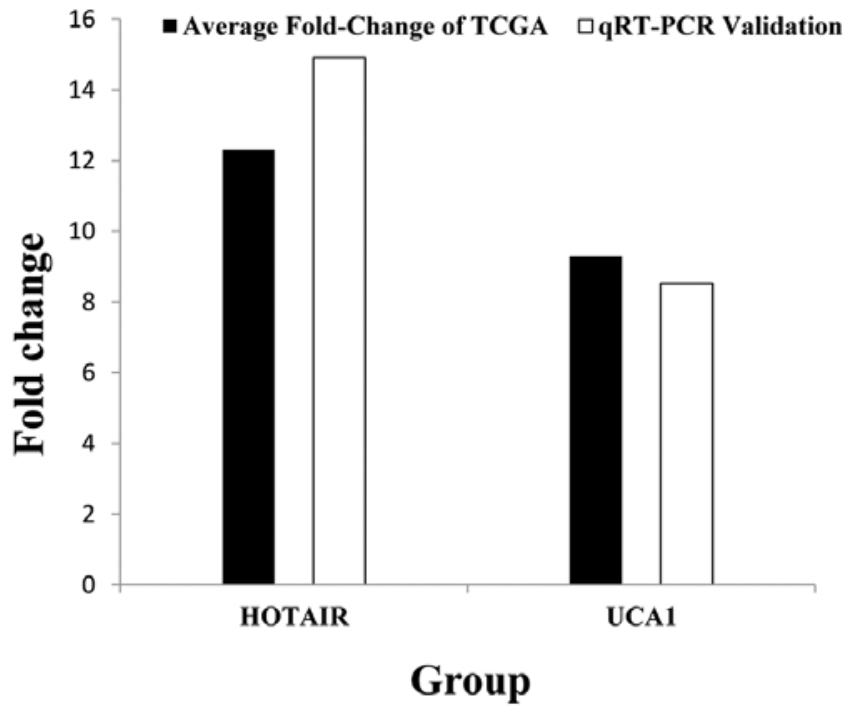

Figure 8. Quantitative RT-PCR validation of two differentially expressed key lncRNAs. Comparison of fold change $\left(2^{-\Delta \Delta C}\right)$ of 1 ncRNAs between TCGA and qRT-PCR results.

the same, and the results showed that our bioinformatics analysis is credible.

\section{Discussion}

Although over several decades there appears to a slight decline in gastric cancer (GC) incidence and associated mortality (27), it still has outstanding incidence and mortality in China, with a large number of patients diagnosed with an advanced stage and poor prognosis (28). Many Japanese series have consistently reported that early diagnosis and treatment of GC, with a 5-year survival rate approximately $90 \%$ (29). However, the diagnosis of gastrointestinal (GI) endoscopy cannot find all precancerous diseases and the early stages GC (30). Therefore, in order to improve this situation, increasing attention has been given to the identification of genes and the exact regulatory mechanism of GC development and progress. Recent years, IncRNAs have been found to be associated with wide range of biological regulatory functions (31). Many studies have reported that IncRNAs participated in pathogenesis of cancers, epigenome, levels of transcription and post-transcription (32-34). To date, only a few studies have reported the expression profiles of lncRNA in GC by microarray or sequencing, and with small sample size (35). LncRNA and mRNA co-expression network was built by significantly differently expressed lncRNA and mRNA (36). In addition, some studies also described interactions between lncRNAs and miRNAs (37-39) or lncRNAs and mRNAs (40) in GC, the results of which showed that lncRNAs may function as a part of GC related regulation network, but lncRNA functions are still poorly explored.

In the present study, we identified tumor grade and lymphatic metastasis related specific lncRNAs, mRNAs and miRNAs in GC from TCGA database. We predicted functions of differentially expressed genes in GC by GO and Pathway analysis. Then, according to the bioinformatics differential analysis we constructed a ceRNA network with tumor grade and lymphatic metastasis related GC specific lncRNAs, mRNAs and miRNAs, which provides an integrated biological views of ceRNA network. Furthermore, we selected key lncRNAs from ceRNA network and further investigated their distributions in different GC clinical features and their correlations with overall survival on the basis of RNA sequencing profile from TCGA. Finally, we randomly selected two key lncRNAs (HOTAIR, UCA1) and analyzed their actual expression levels in the 82 newly diagnosed tumor tissues of GC patients and adjacent non-tumor tissues using qRT-PCR and further analyzed the association between the two key lncRNAs and clinicopathological characteristics to confirm the reliability and validity of the results of the bioinformatics analysis.

Based on the RNA sequence data from TCGA, we found that 25 specific lncRNAs, 22 specific miRNAs and 489 specific lncRNAs were differentially expressed in different tumor stage and lymphatic metastasis of GC patients from $361 \mathrm{GC}$ tumor tissues and 34 non-tumor stomach tissues. Focusing on dysregulation mRNAs through GO and Pathway analysis, the GO results suggested the functions of significant differences in the aspects of immune functions, metabolism and cellular functions, the significant differences in the pathways mainly focus on cancer-related pathways such as 'PI3K-Akt signaling pathway, Wnt signaling pathway, pathways in cancer and small cell lung cancer'. Many pathway-related analysis of the GC significant difference genes, Liu et al (41) and Ren et al (42), also found 'PI3K-Akt signaling pathway and Wnt signaling pathway' were related with GC cell functions.

In addition, many studies have reported that IncRNAs may function as ceRNA regulators to communicate with other RNA transcripts $(15,43-45)$. For example, the lncRNA H19 has been shown to play an important role in tumor progress (46), by acting as an endogenous RNA sponge to inhibit miR-675 in breast cancer and reducing miR-675 mediated translational 

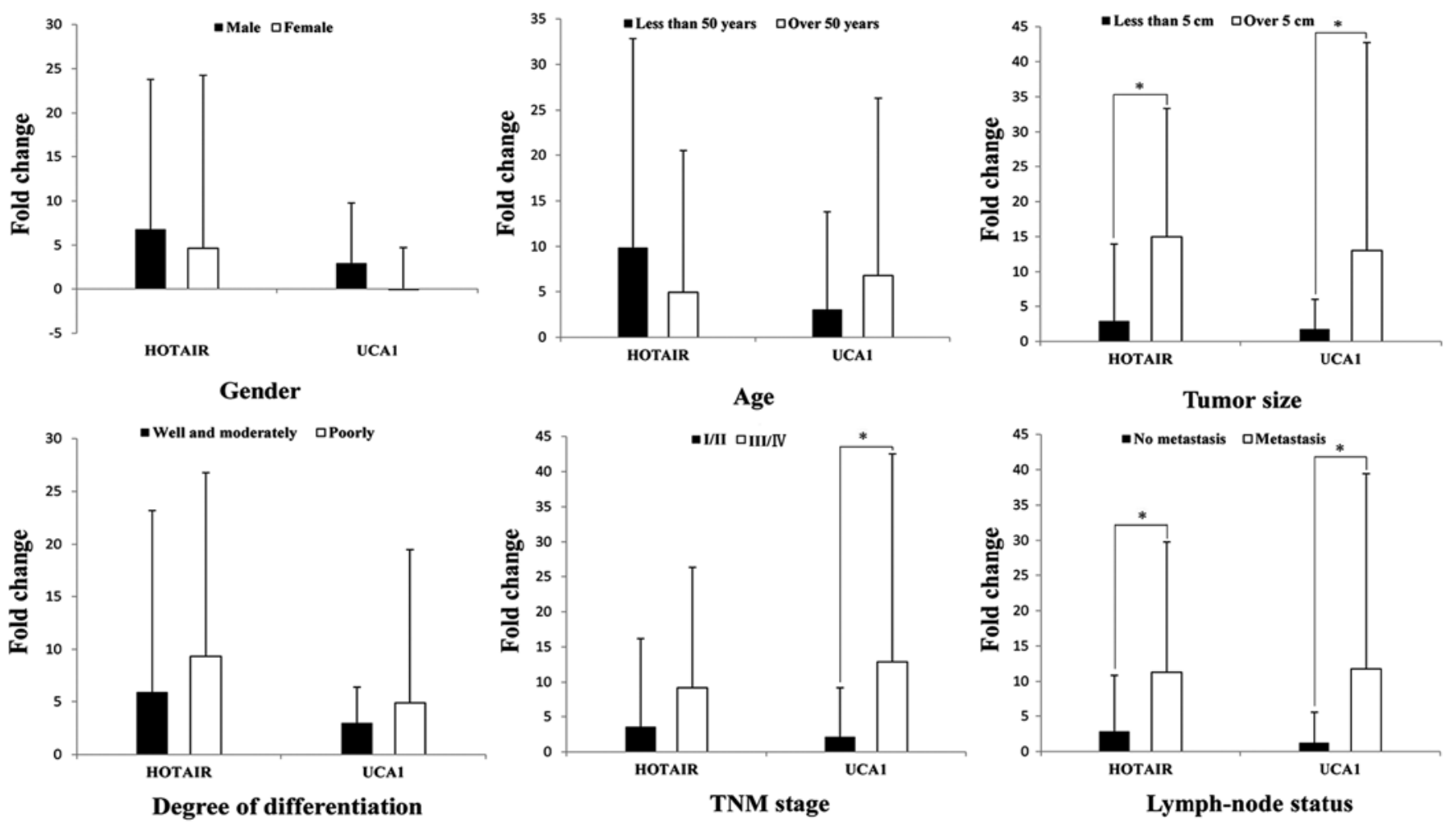

Figure 9. Association between the expression of HOTAIR, UCA1 and clinicopathological characteristics in 82 GC ( $\mathrm{p}<0.05)$.

repression of c-Cb1 and Cb1-b (47). Therefore, there may be some internal contact between IncRNA-miRNA-mRNA in the progress and development of GC. Based on significant differences in IncRNA, miRNA and mRNA expression data, we construction the ceRNA network by bioinformatics prediction and correlation analysis. The ceRNA network we built reveals an unknown ceRNA regulatory network in GC. Recent study also identified that lncRNA interactions with miRNA and mRNA might act as potential diagnosis and prognosis biomarkers in cancer, such as H19, PVT1, HOTAIR, UCA1 and TINCR (7,48-51). In our ceRNA network, we also found these key lncRNAs. Furthermore, many genes from the ceRNA network also were reported as oncogenes and tumor suppressors participating in cancer development and progression, such as COL1A1, FIGF, GHR, HOXC10, LIFR and SF11B (52-54). In the present study, we analyzed the GC specific lncRNA indirectly related mRNAs signal pathways involved in ceRNA network. The pathways analysis results showed that there were four pathways related with cancer. In addition, Ke et al also found that the miR-326 was overexpressed after knockdown lncRNA HOTAIR in glioma cells, which reduced FGF1 expression by activating the PI3K/AKT pathway (55). Therefore, our results suggested that these key IncRNAs may play an important role in the progression and development of GC and the cancer genes related pathways.

To gain insight into the function of lncRNA, 19 key IncRNAs from ceRNA network were analyzed for associations with the clinical features such as gender, race, tumor grade, TNM staging and lymphatic metastasis. The results suggested that 14 lncRNAs were associated with the above indicators, such as HOTAIR, TINCR and H19. These indicators of lncRNAs mainly focus on tumor grade, TNM staging and lymphatic metastasis, among these lncRNAs, H19, and HOTAIR also were reported to be indicators of invasion and lymphatic metastasis of GC $(56,57)$. However, the function of other IncRNAs has not been reported with relevant features yet. Then, we also analyzed the associations between the above 19 key lncRNAs and patient survival. The results showed that eight lncRNAs were related to GC overall survival. Among these eight lncRNAs only H19 has been reported in the survival of hepatocellular carcinoma (58), and the other lncRNAs (RPLP0P2, FOXD2-AS1, TINCR, SLC26A4-AS1, SMIM10L2A, SMIM10L2B and SNORD116-4) were not reported yet. However, the results of lncRNAs and overall survival reveals potential indictors of prognosis in GC.

Subsequently, we randomly selected two key lncRNAs (HOTAIR, and UCA1) and used qRT-PCR validation to confirm the reliability and validity of the above bioinformatics results from the 82 newly diagnosed GC patients. The results from the TCGA and qRT-qPCR experiments were $100 \%$ in agreement. Correlation analysis between the HOTAIR, UCA1 and the clinical features were performed. The results showed that HOTAIR was significantly correlated with tumor size and lymphatic metastasis, and UCA1 was significantly correlated with tumor size, TNM stage and lymphatic metastasis. The two lncRNAs are clinically relevant and the bioinformatics analysis were almost the same, and the results showed that our bioinformatics analysis is credible.

In conclusion, the present study successfully identified cancer specific lncRNAs in GC by bioinformatics analysis from hundreds of candidate lncRNAs and large scale samples in TCGA database. Moreover, we constructed a ceRNA network; it provided a new approach to lncRNA research in GC. Importantly, we analyzed abnormal expression pattern of GC 
specific lncRNAs under different clinical features and overall survival. We also used qRT-PCR validation for the reliability and validity of our bioinformatics analysis results. Results revealed that IncRNAs (ATP8B5P, FOXD2-AS1, GUCY1B2, H19, HOTAIR, LOC100128164, SNORD116-4, LOC553137, PART1, PGM5-AS1, PVT1, RHPN1-AS1, RPLP0P2, SLC26A4-AS1, SMIM10L2A, SMIM10L2B, TINCR, TSPEAR-AS2 and UCA1) may be considered as potential specificity biomarkers in the diagnosis, prognosis and classification of GC.

\section{Acknowledgements}

This study was financially supported by the National Natural Science Foundation of China (81472939, 81172618, 81502783), the Qing Lan Project (no. 2012), the 333 project of Jiangsu Province (no. 2012), the Liu Da Ren Cai Gao Feng Project of Jiangsu Province (no. 2013-WSW-053) and the Fundamental Research Funds for the Central Universities (no. 2014).

\section{References}

1. Guttman M, Amit I, Garber M, French C, Lin MF, Feldser D, Huarte M, Zuk O, Carey BW, Cassady JP, et al: Chromatin signature reveals over a thousand highly conserved large noncoding RNAs in mammals. Nature 458: 223-227, 2009.

2. Kapranov P, St Laurent G, Raz T, Ozsolak F, Reynolds CP, Sorensen PH, Reaman G, Milos P, Arceci RJ, Thompson JF, et al: The majority of total nuclear-encoded non-ribosomal RNA in a human cell is 'dark matter' un-annotated RNA. BMC Biol 8 : $149,2010$.

3. Sana J, Faltejskova P, Svoboda M and Slaby O: Novel classes of non-coding RNAs and cancer. J Transl Med 10: 103, 2012.

4. Kunej T, Obsteter J, Pogacar Z, Horvat S and Calin GA: The decalog of long non-coding RNA involvement in cancer diagnosis and monitoring. Crit Rev Clin Lab Sci 51: 344-357, 2014.

5. Hung T and Chang HY: Long noncoding RNA in genome regulation: Prospects and mechanisms. RNA Biol 7: 582-585, 2010.

6. Pan W, Liu L, Wei J, Ge Y, Zhang J, Chen H, Zhou L, Yuan Q, Zhou C and Yang M: A functional lncRNA HOTAIR genetic variant contributes to gastric cancer susceptibility. Mol Carcinog 55: 90-96, 2016

7. Huang C, Cao L, Qiu L, Dai X, Ma L, Zhou Y, Li H, Gao M, $\mathrm{Li}$ W, Zhang Q, et al: Upregulation of $\mathrm{H} 19$ promotes invasion and induces epithelial-to-mesenchymal transition in esophageal cancer. Oncol Lett 10: 291-296, 2015.

8. Wang F, Xie C, Zhao W, Deng Z, Yang H and Fang Q: Long noncoding RNA CARLo-5 expression is associated with disease progression and predicts outcome in hepatocellular carcinoma patients. Clin Exp Med: Oct 3, 2015 (Epub ahead of print).

9. Zheng HT, Shi DB, Wang YW, Li XX, Xu Y, Tripathi P, Gu WL, Cai GX and Cai SJ: High expression of lncRNA MALAT1 suggests a biomarker of poor prognosis in colorectal cancer. Int J Clin Exp Pathol 7: 3174-3181, 2014

10. Loewen G, Jayawickramarajah J, Zhuo Y and Shan B: Functions of IncRNA HOTAIR in lung cancer. J Hematol Oncol 7: 90, 2014

11. Liu Y, Zhao J, Zhang W, Gan J, Hu C, Huang G and Zhang Y: lncRNA GAS5 enhances G1 cell cycle arrest via binding to YBX1 to regulate p21 expression in stomach cancer. Sci Rep 5: 10159, 2015.

12. Li L, Zhang L, Zhang Y and Zhou F: Increased expression of LncRNA BANCR is associated with clinical progression and poor prognosis in gastric cancer. Biomed Pharmacother 72 : 109-112, 2015.

13. Wang Y, Liu X, Zhang H, Sun L, Zhou Y, Jin H, Zhang H, Zhang H, Liu J, Guo H, et al: Hypoxia-inducible lncRNAAK058003 promotes gastric cancer metastasis by targeting $\gamma$-synuclein. Neoplasia 16: 1094-1106, 2014.

14. Salmena L, Poliseno L, Tay Y, Kats L and Pandolfi PP: A ceRNA hypothesis: The Rosetta Stone of a hidden RNA language? Cell 146: 353-358, 2011.

15. Song X, Cao G, Jing L, Lin S, Wang X, Zhang J, Wang M, Liu W and Lv C: Analysing the relationship between lncRNA and protein-coding gene and the role of lncRNA as ceRNA in pulmonary fibrosis. J Cell Mol Med 18: 991-1003, 2014.
16. Gu W, Gao T, Sun Y, Zheng X, Wang J, Ma J, Hu X, Li J and $\mathrm{Hu}$ M: LncRNA expression profile reveals the potential role of lncRNAs in gastric carcinogenesis. Cancer Biomark 15: 249-258, 2015.

17. Zhang T, Jiang M, Chen L, Niu B and Cai Y: Prediction of gene phenotypes based on GO and KEGG pathway enrichment scores. Biomed Res Int 2013: 870795, 2013.

18. Shannon P, Markiel A, Ozier O, Baliga NS, Wang JT, Ramage D, Amin N, Schwikowski B and Ideker T: Cytoscape: A software environment for integrated models of biomolecular interaction networks. Genome Res 13: 2498-2504, 2003.

19. Vu HL, Troubetzkoy S, Nguyen HH, Russell MW and Mestecky J: A method for quantification of absolute amounts of nucleic acids by (RT)-PCR and a new mathematical model for data analysis. Nucleic Acids Res 28: E18, 2000.

20. Li X, Chen H, Li J and Zhang Z: Gene function prediction with gene interaction networks: A context graph kernel approach. IEEE Trans Inf Technol Biomed 14: 119-128, 2010.

21. Hao NB, Tang B, Wang GZ, Xie R, Hu CJ, Wang SM, Wu YY, Liu E, Xie X and Yang SM: Hepatocyte growth factor (HGF) upregulates heparanase expression via the PI3K/Akt/NF- $\kappa \mathrm{B}$ signaling pathway for gastric cancer metastasis. Cancer Lett 361: 57-66, 2015.

22. Pan KF, Liu WG, Zhang L, You WC and Lu YY: Mutations in components of the Wnt signaling pathway in gastric cancer. World J Gastroenterol 14: 1570-1574, 2008.

23. Chatzinikolaou G, Nikitovic D, Stathopoulos EN, Velegrakis GA, Karamanos NK and Tzanakakis GN: Protein tyrosine kinase and estrogen receptor-dependent pathways regulate the synthesis and distribution of glycosaminoglycans/proteoglycans produced by two human colon cancer cell lines. Anticancer Res 27: 4101-4106, 2007.

24. Di J, Huang H, Qu D, Tang J, Cao W, Lu Z, Cheng Q, Yang J, Bai J, Zhang Y, et al: Rap2B promotes proliferation, migration, and invasion of human breast cancer through calcium-related ERK1/2 signaling pathway. Sci Rep 5: 12363, 2015.

25. Jeggari A, Marks DS and Larsson E: miRcode: A map of putative microRNA target sites in the long non-coding transcriptome. Bioinformatics 28: 2062-2063, 2012.

26. Hsu SD, Tseng YT, Shrestha S, Lin YL, Khaleel A, Chou CH, Chu CF, Huang HY, Lin CM, Ho SY, et al: miRTarBase update 2014: An information resource for experimentally validated miRNA-target interactions. Nucleic Acids Res 42: D78-D85, 2014.

27. Patru CL, Surlin V, Georgescu I and Patru E: Current issues in gastric cancer epidemiology. Rev Med Chir Soc Med Nat Iasi 117: 199-204, 2013.

28. Li G, Hu Y and Liu H: Current status of randomized controlled trials for laparoscopic gastric surgery for gastric cancer in China. Asian J Endosc Surg 8: 263-267, 2015.

29. Tanaka N, Katai H, Taniguchi H, Saka M, Morita S, Fukagawa T and Gotoda T: Trends in characteristics of surgically treated early gastric cancer patients after the introduction of gastric cancer treatment guidelines in Japan. Gastric Cancer 13: 74-77, 2010.

30. Rajan E, Gostout CJ, Aimore Bonin E, Moran EA, Locke RG, Szarka LA, Talley NJ, Deters JL, Miller CA, Knipschield MA, et al: Endoscopic full-thickness biopsy of the gastric wall with defect closure by using an endoscopic suturing device: Survival porcine study. Gastrointest Endosc 76: 1014-1019, 2012.

31. Moran VA, Perera RJ and Khalil AM: Emerging functional and mechanistic paradigms of mammalian long non-coding RNAs. Nucleic Acids Res 40: 6391-6400, 2012.

32. Gutschner T and Diederichs S: The hallmarks of cancer: A long non-coding RNA point of view. RNA Biol 9: 703-719, 2012.

33. Zhang H, Chen Z, Wang X, Huang Z, He Z and Chen Y: Long non-coding RNA: A new player in cancer. J Hematol Oncol 6: 37, 2013.

34. Kornienko AE, Guenzl PM, Barlow DP and Pauler FM: Gene regulation by the act of long non-coding RNA transcription. BMC Biol 11: 59, 2013.

35. Song H, Sun W, Ye G, Ding X, Liu Z, Zhang S, Xia T, Xiao B, $\mathrm{Xi} \mathrm{Y}$ and Guo J: Long non-coding RNA expression profile in human gastric cancer and its clinical significances. J Transl Med 11: $225,2013$.

36. Lin XC, Zhu Y, Chen WB, Lin LW, Chen DH, Huang JR, Pan K, Lin Y, Wu BT, Dai Y, et al: Integrated analysis of long non-coding RNAs and mRNA expression profiles reveals the potential role of lncRNAs in gastric cancer pathogenesis. Int $\mathbf{J}$ Oncol 45: 619-628, 2014. 
37. Zhou X, Ye F, Yin C, Zhuang Y, Yue G and Zhang G: The interaction between miR-141 and lncRNA-H19 in regulating cell proliferation and migration in gastric cancer. Cell Physiol Biochem 36: 1440-1452, 2015.

38. Chen X: Predicting lncRNA-disease associations and constructing lncRNA functional similarity network based on the information of miRNA. Sci Rep 5: 13186, 2015.

39. Peng W, Si S, Zhang Q, Li C, Zhao F, Wang F, Yu J and Ma R: Long non-coding RNA MEG3 functions as a competing endogenous RNA to regulate gastric cancer progression. J Exp Clin Cancer Res 34: 79, 2015.

40. Kong R, Zhang EB, Yin DD, You LH, Xu TP, Chen WM, Xia R, Wan L, Sun M, Wang ZX, et al: Long noncoding RNA PVT1 indicates a poor prognosis of gastric cancer and promotes cell proliferation through epigenetically regulating p15 and p16. Mol Cancer 14: 82, 2015.

41. Liu M, Li CM, Chen ZF, Ji R, Guo QH, Li Q, Zhang HL and Zhou YN: Celecoxib regulates apoptosis and autophagy via the PI3K/Akt signaling pathway in SGC-7901 gastric cancer cells. Int J Mol Med 33: 1451-1458, 2014.

42. Ren X, Zheng D, Guo F, Liu J, Zhang B, Li H and Tian W: PPAR $\gamma$ suppressed Wnt/ $\beta$-catenin signaling pathway and its downstream effector SOX9 expression in gastric cancer cells. Med Oncol 32: 91, 2015.

43. Wu Q, Guo L, Jiang F, Li L, Li Z and Chen F: Analysis of the miRNA-mRNA-lncRNA networks in $\mathrm{ER}^{+}$and $\mathrm{ER}^{-}$breast cancer cell lines. J Cell Mol Med 19: 2874-2887, 2015.

44. Hu Y, Tian H, Xu J and Fang JY: Roles of competing endogenous RNAs in gastric cancer. Brief Funct Genomics pii: elv036, Sep 24, 2015 (Epub ahead of print).

45. Zhang J, Fan D, Jian Z, Chen GG and Lai PB: Cancer specific long noncoding RNAs show differential expression patterns and competing endogenous RNA potential in hepatocellular carcinoma. PLoS One 10: e0141042, 2015.

46. Raveh E, Matouk IJ, Gilon M and Hochberg A: The H19 Long non-coding RNA in cancer initiation, progression and metastasis - a proposed unifying theory. Mol Cancer 14: 184, 2015.

47. Vennin C, Spruyt N, Dahmani F, Julien S, Bertucci F, Finetti P, Chassat T, Bourette RP, Le Bourhis X and Adriaenssens E: H19 non coding RNA-derived miR-675 enhances tumorigenesis and metastasis of breast cancer cells by downregulating c-Cbl and Cbl-b. Oncotarget 6: 29209-29223, 2015.

48. Liu XH, Sun M, Nie FQ, Ge YB, Zhang EB, Yin DD, Kong R, Xia R, Lu KH, Li JH, et al: Lnc RNA HOTAIR functions as a competing endogenous RNA to regulate HER 2 expression by sponging miR-331-3p in gastric cancer. Mol Cancer 13: 92, 2014.
49. Ding J, Li D, Gong M, Wang J, Huang X, Wu T and Wang C: Expression and clinical significance of the long non-coding RNA PVT1 in human gastric cancer. Onco Targets Ther 7: 1625-1630, 2014.

50. Zheng Q, Wu F, Dai WY, Zheng DC, Zheng C, Ye H, Zhou B, Chen JJ and Chen P: Aberrant expression of UCA1 in gastric cancer and its clinical significance. Clin Transl Oncol 17: 640-646, 2015.

51. Xu TP, Liu XX, Xia R, Yin L, Kong R, Chen WM, Huang MD and Shu YQ: SP1-induced upregulation of the long noncoding RNA TINCR regulates cell proliferation and apoptosis by affecting KLF2 mRNA stability in gastric cancer. Oncogene 34: 5648-5661, 2015.

52. Li AQ, Si JM, Shang Y, Gan LH, Guo L and Zhou TH: Construction of COL1A1 short hairpin RNA vector and its effect on cell proliferation and migration of gastric cancer cells. Zhejiang Da Xue Xue Bao Yi Xue Ban 39: 257-263, 2010 (In Chinese).

53. Ran G, Lin Y, Cao P, Cai XT and Li SY: Effect of rhGH on JAK2-STAT3 signal pathway after GHR was down-regulated by siRNA in gastric cancer cell. Yao Xue Xue Bao 48: 435-440, 2013 (In Chinese).

54. Feng X, Li T, Liu Z, Shi Y and Peng Y: HOXC10 up-regulation contributes to human thyroid cancer and indicates poor survival outcome. Mol Biosyst 11: 2946-2954, 2015.

55. Ke J, Yao YL III, Zheng J, Wang P, Liu YH, Ma J, Li Z, Liu XB, Li ZQ, Wang ZH, et al: Knockdown of long non-coding RNA HOTAIR inhibits malignant biological behaviors of human glioma cells via modulation of miR-326. Oncotarget 6 : 21934-21949, 2015.

56. Li H, Yu B, Li J, Su L, Yan M, Zhu Z and Liu B: Overexpression of lncRNA H19 enhances carcinogenesis and metastasis of gastric cancer. Oncotarget 5: 2318-2329, 2014.

57. Zhang ZZ, Shen ZY, Shen YY, Zhao EH, Wang M, Wang CJ, $\mathrm{CaO} \mathrm{H}$ and $\mathrm{Xu}$ J: HOTAIR long noncoding RNA promotes gastric cancer metastasis through suppression of Poly $\mathrm{r}(\mathrm{C})$-Binding Protein (PCBP) 1. Mol Cancer Ther 14: 1162-1170, 2015.

58. Yang Z, Lu Y, Xu Q, Tang B, Park CK and Chen X: HULC and H19 played different roles in overall and disease-free survival from hepatocellular carcinoma after curative hepatectomy: a preliminary analysis from gene expression omnibus. Dis Markers 2015: 191029, 2015. 